\title{
Development of Syllabus Feature Extraction System
}

\author{
Kazuhide Sugimoto ${ }^{1}$ Kentaro Noguchi $^{1}$ Chikatoshi Yamada ${ }^{1}$ Takahiro \\ Yonamine $^{2}$ \\ ${ }^{1}$ Okinawa National College of Technology \\ ${ }^{2}$ Sendai National College of Technology
}

\begin{abstract}
In order to guarantee the quality of the student's skill level and the acquisition level of knowledge, we have developed the embedded technology skill standards for national colleges of technology in Japan based on syllabus data. In this paper, the function and implementation of a syllabus feature extraction system are reported. By using the system, what kinds of learning items and skills which can be learned and mastered by students by choosing a subject are clarified by comparing with "skill sheet" that defines skill standards. The system has already been implemented on a cloud server for verification of its performance.
\end{abstract}

Keywords: Syllabus, Educational curriculum, Skill standards, Learning management system

\section{Introduction}

Recently, demands for skillful engineers for developing higher application of embedded systems increase in the industry [1]. In both universities and national colleges of technology, the higher education for embedded technology skill has been practiced in Japan. However, the systematization of the curriculum for embedded skill training is an item to be solved. We worked on a project and developed skill standards with both educational contents and the new curriculum in cooperation with 10 national colleges of technology (NCTs) [2].

Embedded technology skill standards have been designed as a "skill sheet", and based on the standards, mastered skills by each student can be visualized on a skill management system which is linked to learning management system (LMS) to encourage their learning motivation.

Educational programs in NCTs are mainly for fundamental skills and basic knowledge through experiments. Therefore, it is very important to clarify the correspondences, in other words a gap, between the demanded engineer's skills in industry and student's ones in NCTs, and to define embedded technology skill standards for evaluation.

\section{Syllabus and skill standards}

\subsection{Syllabus}

A syllabus is provided as an outline and summary of topics and specific information about each subject to clarify the objectives and the items which should be learned and mastered with a schedule of test dates and the grading policy and etc. Fig. 1 shows an example of syllabus.

\subsection{Skill standards}

In order to guarantee student's skill level, it is necessary to develop standard educational contents and skill check system. 


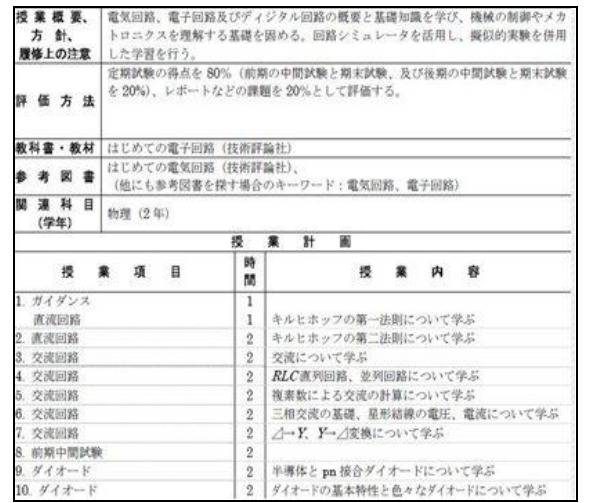

Fig. 1: An example of syllabus (in Japanese)

For this purpose, we have defined embedded technology skill standards by comparing and combining all educational contents and programs in NCTs to extract fundamental skills by keywords with tags to each learning item. Here, feature extraction modules play a significant role and they should be implemented as server programs that can be used via internet from all NCTs. By using these modules, each skill which should be learned and mastered by each student can be extracted automatically from each subject's syllabus data which is introduced for the learning plan summary of lectures and/or engineering experiments. Then, extracted skills are categorized and mapped into a "skill sheet". Fig.2 shows its framework.

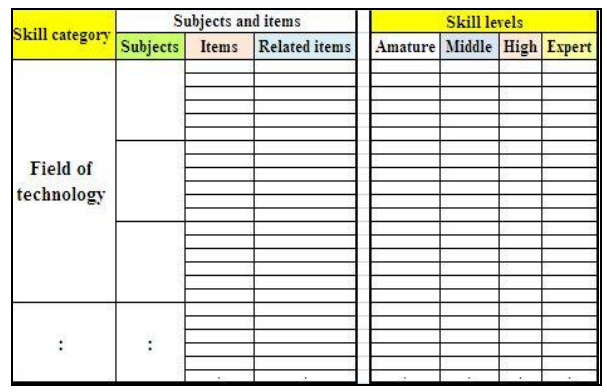

Fig. 2: "Skill sheet" framework

The standard skills are managed with three hierarchical classes a 'skill sheet'.
They are skill categories, subjects and items. And each item is classified in conjunction with related items in the same class. Each student's skill level can be classified in four levels by the score of an achievement test as shown in Fig.3. Each student can check own skill level and its growth, now we are also developing standard examination by collaborating with an outside certification organization.

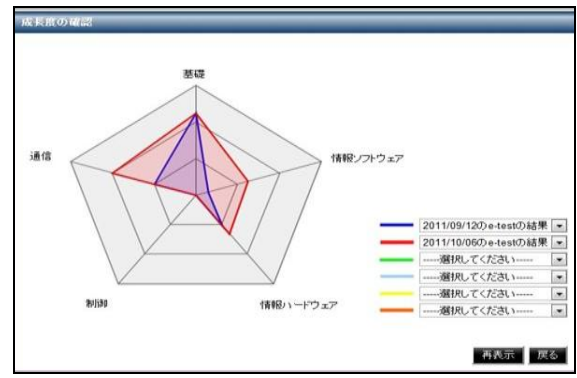

Fig. 3: Mastered skill level of a student

Based on a 'skill sheet', characteristics of curriculums between NCTs can also be displayed and compared on a chart as shown in Fig.4 [3].

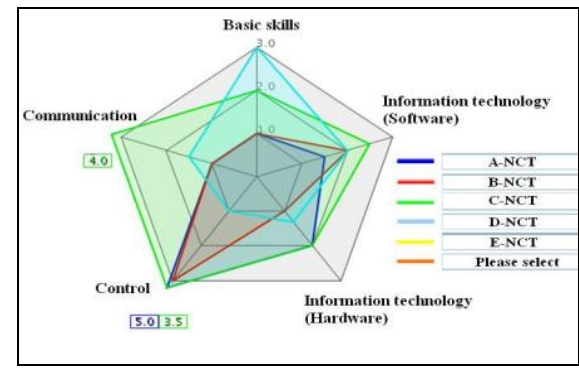

Fig. 4: Characteristics of curriculums by comparison of skill levels between NCTs

\section{Syllabus feature extraction system}

\subsection{Functions}

As mentioned above, in order to extract characteristics of curriculums, each characteristic of related subjects has to be ex- 
tracted from syllabus data by checking corresponding items with "skill sheet". For this purpose, we have developed a system for extracting characteristics of subject that we call syllabus feature extraction system (SFE).

Extracted data by SFE is structured hierarchically according to the one of "skill sheet", in other words, has the same structure as "skill sheet" that is composed of "skill categories", "standard subjects", "learning items", with "related items". However, before the extraction, each learning item has to be set into a database file that can be used as a dictionary for comparison to check correspondence between items in a "skill sheet" and input syllabus data. Now, we use a "skill sheet" as a dictionary. Of course, SFE can handle other type of files as a dictionary that should be provided as a database file such as CSV (comma-separated values) format file and user can specify the file to SFE for changing the dictionary file. As for handling Japanese characters, character code set of input file is limited to ShiftJIS code by the current version of SFE.

Extraction process is proceeded by the following steps.

- Input syllabus file is converted into a plain text file.

- For each item in the input file, by comparing with the dictionary file, check the corresponding item's existence. If exists, the item is extracted.

- For all of the items in the input file, above procedure is repeated and all of the corresponding items are listed up for output.

After extraction, extracted items are formatted into the same structure as "skill sheet" and output as CVS formatted file. Here, each item can be saved with link information to both of the related field and standard subject based on the same structure as "skill sheet" or the dictionary file.
In consideration of both simplification of procedure and processing speed, this correspondence search process is implemented as the exact match by comparing each input item in the input syllabus file with all of the items in the dictionary file. So, when the exact match can be found, it is determined that the item is included in input syllabus file. As for the input file, the following file formats are supported.

- Microsoft ${ }^{\circledR}$ Word

- Microsoft $\AA$ Excel

- Microsoft巴 PowerPoint

- PDF

- Plain text

Document format is with no limitation, user can also input a lecture document, for example, as input instead of the syllabus file. Fig. 5 shows a menu window of SFE. There are five buttons. From top to the bottom, plain text conversion, matching with dictionary, output formatter, batch processing, and environment setting, respectively.

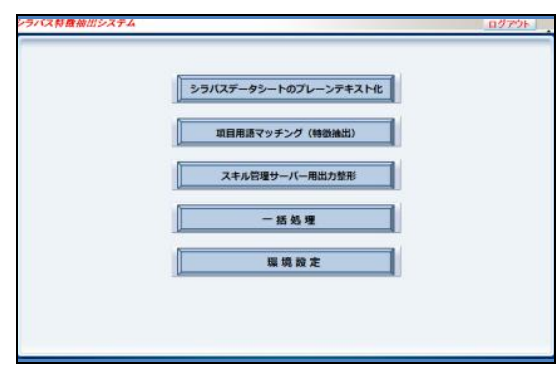

Fig. 5: Menu window of SFE (in Japanese)

And Fig.6 shows operation window for plain text conversion mode of SFE. Users can input a syllabus data file on the local machine into the server by put the filename into the upper input area. Here, multiple file input by using ZIP archived file is also supported. And user can specify the output filename with its location for the file should be saved on the local machine. Fig.7 shows an example of the output file. The name of the college and department with the year are also added. 


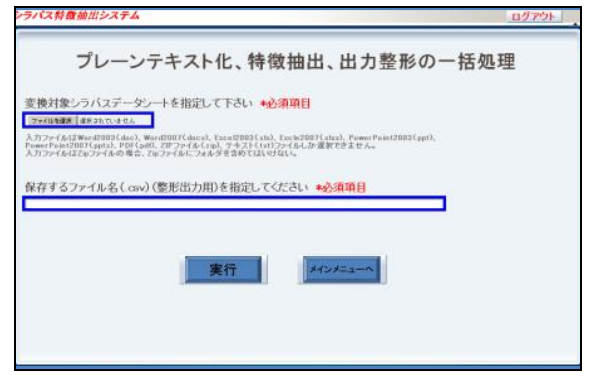

Fig. 6: Operation window for plain text conversion mode of SFE (in Japanese)

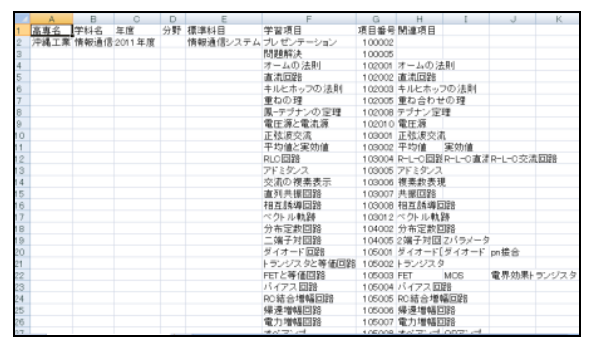

Fig. 7: An example of the output file (in Japanese)

\subsection{Implementation}

Since SFE service has to be used by all of the members in registered NCTs at any time, SFE has been implemented on a cloud server as server side programs that can be used via internet. Now, it is implemented on Amazon Elastic Compute Cloud (Amazon EC2) [4].

- CPU: Intel Xeon $(2.67 \mathrm{GHz})$

- Memory: 4GB (PC3-10600)

- HDD: 250GB SATA (RAID-5)

- OS: CentOS 5.6 (32bit)

Modules: Java1.6.0, Apache2.2.19, Tomcat6.0.29(security-patched), MySQL5.1.57

\section{Conclusion}

In this paper, functions and implementation of the syllabus feature extraction system (SFE) are reported. SFE has been implemented on a cloud server as server side programs that can be used by registered NCTs via internet. By using these modules, each skill which should be learned and mastered by each student can be extracted automatically from each subject's syllabus data which is introduced for the learning plan summary of lectures and/or engineering experiments. Then, extracted skills are categorized and mapped into a 'skill sheet'.

Based on a "skill sheet" that we have defined as an embedded technology skill standard based on syllabus data for about 500 subjects gathering from 10 NCTs, the skill levels of all students can be extracted and visualized. In addition to the above, by comparing skill levels of students, curriculum characteristics can also be extracted and it is used not only for its improvement but faculty development of teaching staffs.

Now, we are trying to extend a "skill sheet" to the one which can be used for handling and managing skills for engineering experiments. Furthermore, to define the core curriculum that describes common minimum standard about learning contents, and the standard that should be acquired by students.

\section{References}

[1] "Summary of Embedded Software Industry Survey." 2008. meti.go.jp. http://www.meti.go.jp/english/press/d ata/nBackIssue20080522_01.html

[2] “K-skill.” 2009. sendai-nct.ac.jp. http://hirose.sendai-nct.ac.jp/renkeigp/english.html.

[3] K. Sugimoto, K. Noguchi, C. Yamada and T. Yonamine, "Development and Practice of National Colleges of Technology Version Embedded Technology Skill Standards," Proc. 41 st ASEE/IEEE Frontiers in Education Conf., TC1-4, 2011.

[4] http://aws.amazon.com/ec2/ 